\title{
Neighborhood consistency and memory for number facts
}

\author{
Jamie I. D. Campbell • Roxanne R. Dowd • \\ Jillian M. Frick • Kendra N. McCallum • \\ Arron W. S. Metcalfe
}

Published online: 6 January 2011

(C) Psychonomic Society, Inc. 2010

\begin{abstract}
Verguts and Fias (Memory \& Cognition 33:1-16, 2005a) proposed a new model of memory for simple multiplication facts $(2 \times 3=6 ; 8 \times 7=56)$ in which learning and performance is governed by the consistency of a problem's correct product with neighboring products in the times table. In the present study, to directly investigate effects of neighborhood consistency, participants memorized a set of 16 novel "pound" arithmetic equations. The pound arithmetic table included eight tie equations with repeated operands (e.g., 4 \# $4=29$ ) and eight nontie equations (e.g., 5 \# 4 =39). In the consistent problem set, tie and nontie answers in adjacent columns and rows shared a common decade or unit value. In the inconsistent problem set, neighboring tie and nontie problems did not share a common decade or unit. Across 14 study-test blocks, memorization of the pound arithmetic table presented a robust effect of neighborhood consistency, with the rate of learning nearly doubling that of the inconsistent condition. An analysis of error types showed that consistency fostered the development of a categorical structure based on problem operands and that tie problems were encoded as a distinct subcategory of problems. There was also a substantial learning advantage for tie problems relative to nonties both with consistent and inconsistent neighbors. The results indicate that neighborhood consistency can have a major impact on memory for number facts.
\end{abstract}

\footnotetext{
J. I. D. Campbell $(\bowtie) \cdot$ R. R. Dowd J. M. Frick •

K. N. McCallum • A. W. S. Metcalfe

Department of Psychology, University of Saskatchewan,

9 Campus Drive,

Saskatoon, SK S7N 5A5, Canada

e-mail: Jamie.campbell@usask.ca
}

Keywords Memory Retrieval practice $\cdot$ Arithmetic . Number facts

Learning and performance of the basic 1-digit $\times 1$-digit multiplication facts (e.g., $2 \times 3=6 ; 8 \times 4=32$ ) have been studied systematically for more than 80 years (see, e.g., Norem \& Knight, 1930). This effort has been motivated by the fact that elementary arithmetic is a fundamental intellectual skill, and that understanding the underlying memory structures and how they develop during learning could contribute to more effective educational strategies. Verguts and Fias (2005a) introduced a new model of memory for the multiplication facts. Like in previous models (see Verguts \& Fias, 2005a, for a review), they assumed that multiplication facts are represented in a network structure wherein problem operands (the factors) constitute semantic categories, and factor multiples (the products) are category exemplars that are activated when problem operands are presented. The correct product can be retrieved from the network because it is semantically related to both factors and has a history of association with a specific factor pair. Their model, however, introduced a critical new assumption: Whether or not a product shares a common decade or unit value with neighboring products in the times table has a major impact on learning and performance. This neighborhood consistency assumption appears to provide a simple and elegant explanation for diverse phenomena of multiplication memory. Nonetheless, previous research has provided no direct evidence of neighborhood consistency effects. We first review the Verguts and Fias (2005a) interacting neighbors (IN) model and the evidence supporting it. We then report an experiment in which neighborhood consistency was directly manipulated to test a variety of predictions related to their model. 


\section{The interacting neighbors model}

To understand the Verguts and Fias (2005a) model, it is helpful to depict their representational assumptions in terms of a multiplication half table (see Table 1). In Table 1, the columns correspond to the minimum operand, and the rows correspond to the maximum operand with the correct products located at the intersections. Operands range from 2 to 9 , excluding 0 and 1 problems, which are presumed to be solved by rule-based strategies (i.e., $N \times 0=0$; $N \times 1=N$; Ashcraft, 1992; Campbell \& Metcalfe, 2007). Problems with repeated operands $(7 \times 7 ; 8 \times 8$, etc. $)$ are called "tie" problems and appear along the diagonal in Table 1. Much research has found ties to be easier than nontie problems composed of two different operands (e.g., $8 \times 7 ; 8 \times 9$, etc.; see, e.g., Campbell \& Gunter, 2002). With respect to nontie problems (the off-diagonal problems in Table 1), people encounter and solve these problems in both orders (e.g., $7 \times 8$ and $8 \times 7$ ), but the IN model assumes that normally only one order is represented in memory. This assumption is supported by nearly perfect transfer of practice between the two orders (Rickard, 2005; Rickard, Healy, \& Bourne, 1994), which implies that both orders access a common memory representation (but see Robert \& Campbell, 2008).

The fundamental new assumption about multiplication memory introduced in the IN model is that learning and performance of the problems in Table 1 are largely governed by the decade and unit consistency of the correct product with other products in the immediate neighborhood. Specifically, problem operands are assumed to activate operand-related answers surrounding the correct product (Galfano, Rusconi, \& Umiltà, 2003; Phenix \& Campbell, 2004). The accessibility of the correct product depends on its decade and unit consistency with other products in the activated neighborhood. Verguts and Fias (2005b) defined a problem's neighborhood to include only

Table 1 Multiplication half table

\begin{tabular}{lllllllll}
\hline \multicolumn{1}{c}{ Minimum Operand } \\
\cline { 2 - 8 } Maximum Operand & 2 & 3 & 4 & 5 & 6 & 7 & 8 & 9 \\
\hline 2 & $\mathbf{0 4}$ & & & & & & & \\
3 & 06 & $\mathbf{0 9}$ & & & & & & \\
4 & 08 & 12 & $\mathbf{1 6}$ & & & & & \\
5 & 10 & 15 & 20 & $\mathbf{2 5}$ & & & & \\
6 & 12 & 18 & 24 & 30 & $\mathbf{3 6}$ & & & \\
7 & 14 & 21 & 28 & 35 & 42 & $\mathbf{4 9}$ & & \\
8 & 16 & 24 & 32 & 40 & 48 & 56 & $\mathbf{6 4}$ & \\
9 & 18 & 27 & 36 & 45 & 54 & 63 & 72 & $\mathbf{8 1}$ \\
\hline
\end{tabular}

Answers to tie problems in bold answers in immediately adjacent columns and rows (e.g., 20 and 30 are immediate neighbors of 25 in Table 1), although Verguts and Fias (2005a) also allowed neighbors that were two columns or rows removed (e.g., 15 and 35) to contribute slightly to answer competition. Each product (e.g., 35) is assumed to be represented by distinct decade (3) and unit (5) components (Nuerk, Weger, \& Willmes, 2001; Ratinckx, Brysbaert, \& Fias, 2005), and the two components contribute independently and equally to neighborhood consistency. Each consistent decade or unit neighbor contributes an increment to consistency, whereas each inconsistent decade or unit neighbor contributes a decrement to consistency. The net contributions of consistent and inconsistent neighbors defines a given product's consistency, with greater consistency (or, equivalently, less inconsistency) leading to faster learning and better memory performance. Consequently, relative differences in neighborhood consistency across problems should predict corresponding differences in memory performance.

Verguts and Fias (2005a) implemented the IN theory in a series of connectionist simulation models. The simulations reproduced several fundamental features of memory for multiplication facts. First, learning and performance were better for small-number problems than for large-number problems. This problem-size effect is a virtually ubiquitous finding in both children's and adult's simple arithmetic (Zbrodoff \& Logan, 2005). The problem-size effect has been attributed to greater associative strength for smallthan for large-number problems, owing to higher practice frequencies (Koshmider \& Ashcraft, 1991; McCloskey \& Lindemann, 1992), greater associative interference for large problems (Campbell, 1995), and greater use of relatively inefficient procedural strategies for large problems (LeFevre et al. 1996; Siegler, 1988). In the IN model, a problem-size effect occurs because small number problems generally have greater neighborhood consistency than do large number problems, mainly because small problems have higher decade consistency (i.e., they share a decade value of 0 or 1). Thus, the IN model implies that the problem-size effect arises from the incidental answer-consistency structure that resides within the multiplication times table.

The IN simulations also reproduced two other prominent features of simple multiplication retrieval performance. For both children and adults, five-times problems $(2 \times 5 ; 3 \times 5$, etc.) are fast and accurate relative to their problem-size, and tie problems (i.e., those with repeated operands such as $2 \times 2 ; 3 \times 3$, etc.) are fast and accurate relative to nonties $(2 \times 3 ; 3 \times 4$, etc.) (e.g., Campbell \& Graham, 1985). Various explanations have been offered for both effects (see Verguts \& Fias, 2005a, for a review). For example, Siegler (1988) proposed that the five-times advantage arises because children learn early on to count by fives, which facilitates solving five-times problems and reinforces their 
memory strength. Campbell (1995) proposed that five-times problems form a distinct categorical subset of problems, which partially insulates them from associative interference. Campbell (1995) also suggested that tie problems similarly constitute a distinct subcategory of problems that encounter relatively less interference from nonties in the same times table. In support of this suggestion, Campbell (1995) pointed out that at least one study (Campbell \& Graham, 1985) showed that errors on five-times problems tended to be answers to other five-times problems (e.g., $5 \times 7=30$ ), and similarly that tie errors tended to be answers to other ties (e.g., $8 \times 8=81$ ). A predominance of within-category errors would support the idea that fives and ties constitute distinct subsets of problems within the multiplication network. Verguts and Fias (2005a) pointed out, however, that other corpora of errors (e.g., Campbell, 1994) did not replicate this pattern for ties.

In the IN model, both the fives and ties effects occur because of neighborhood consistency. Fives always have neighbors within \pm 2 rows/columns in the times table that share a consistent unit value (i.e., 0 or 5), which elevates their neighborhood consistency relative to that of many other problems. The ties lie on the diagonal and therefore have fewer neighbors than do nonties. This affords a tie advantage because, in general, neighbors introduce more competition (inconsistency) than cooperation (consistency). Thus, again, the IN model could explain major features of performance as arising from the inherent neighborhood consistency of the multiplication half table.

The IN model also reproduced prominent features of multiplication errors. Most multiplication errors are operandrelated products (i.e., a multiple of one of the factors), usually the answer to an adjacent problem in the times table (e.g., $8 \times 4=36$ or 24; Campbell, 1995). An increasing proportion of operand-related errors emerges gradually during early schooling (Campbell \& Graham, 1985), and operand-related errors predominate adults' multiplication errors produced under time pressure (Campbell, 1995). The emergence and persistence of operand-related errors reflect the development and consolidation of the factor-based categorical structure of the memory network for multiplication facts (Campbell \& Graham, 1985). The IN model similarly produced a predominance of operand-related errors, and the proportion increased across learning epochs.

\section{Evidence for neighborhood consistency effects}

The preceding paragraphs served to introduce the central concept of neighborhood consistency in the IN model and to outline how this simple assumption potentially accounts for major features of multiplication learning and performance (see Verguts \& Fias, 2005a, for a detailed descrip- tion of the model and the full range of effects it reproduces). Instead of diverse explanations for the problem size, fives, and ties effects in multiplication, the model provides the parsimony of a single theoretical mechanism to explain all of these phenomena.

Nonetheless, although the IN simulations are impressive, they do not provide direct evidence for effects of neighborhood consistency in number-fact memory. To this end, Verguts and Fias (2005b) conducted an experiment to manipulate consistency in simple multiplication.

They reasoned that practicing a specific problem (e.g., $8 \times 8=64$ ) should increase answer competition for neighbor problems with inconsistent answers (e.g., $8 \times 9=72$ ) and disrupt their performance. As predicted, response time (RT) increased when certain problems' neighbors were practiced as compared with when they were not practiced. Subsequently, Domahs, Delazer and Nuerk (2006) analyzed the adult multiplication error data presented in Campbell (1997) to look for effects of neighborhood consistency predicted by the IN model. They concluded that operandrelated errors were more likely to involve decade-consistent answers than decade-inconsistent answers, apparently supporting the IN model. Furthermore, a regression analysis of the error rates across problems indicated that with neighborhood consistency and also the fives and ties effects controlled statistically, there remained little evidence of a problem-size effect on multiplication errors. Domahs et al. concluded that this supports the IN model's assumption that neighborhood consistency contributes to the problem-size effect in simple multiplication.

Although these results potentially indicate consistency effects, there are limitations to both of these studies. The Domahs et al. (2006) study did not directly manipulate neighborhood consistency; consequently, there is no basis to conclude that consistency was the causal factor. Similarly, although Verguts and Fias (2005b) intended to manipulate neighborhood consistency by including or excluding neighboring problems, this would also function to manipulate retrieval-induced forgetting. Phenix and Campbell (2004) showed that practicing a subset of multiplication problems interfered with memory performance for other problems in the same times table, thereby demonstrating that multiplication retrieval is subject to retrieval-induced forgetting, which could account for the Verguts and Fias (2005b) findings. Consequently, there has yet to be clear experimental evidence for neighborhood consistency effects in number-fact retrieval.

\section{The present experiment}

To directly investigate neighborhood consistency in number-fact retrieval, we constructed two sets of novel "pound" 
arithmetic equations. Each problem set consisted of 16 equations with digits 2 through 9 as operand pairs. Each set included eight tie equations (e.g., 4 \# 4 = 29) and eight nontie equations (e.g., 5 \# 4 =39). In the consistent problem set, tie and nontie answers in adjacent columns and rows shared a common decade or unit value. In the inconsistent problem set, answers to neighboring tie and nontie problems did not share a common decade or unit. One group of participants was asked to memorize the consistent-neighborhood problem set and another to memorize the inconsistent-neighborhood problem set. Participants received a series of study-test cycles in which they studied each of the 16 equations one at a time in random order for $3 \mathrm{~s}$ and typed in the correct answer. Then, after $10 \mathrm{~s}$ of counting backward by threes from a given number, they were tested on their ability to recall the correct answer to each pound problem given only the operands (e.g., 5 \# 4 = ?). There were 14 such study-test cycles, which allowed us to track memorization of the pound equations under consistent and inconsistent neighborhood conditions.

This design tested several predictions of the IN model with respect to effects of neighborhood consistency. First, as learning progresses, a performance advantage should emerge for the consistent neighborhood condition relative to the inconsistent neighborhood. Second, we expected the proportion of errors involving neighbors to increase with practice, but if such errors are promoted by neighborhood consistency, as was proposed by Domahs et al. (2006), then neighbor errors should be promoted in the consistent condition relative to the inconsistent condition. Furthermore, if the advantage for tie problems depends on neighborhood consistency, then no tie effect is expected because ties and nonties have equivalent answer consistency in the consistent and inconsistent problem sets. Finally, if the tie advantage occurs because ties form a distinct subcategory of problems (Campbell, 1995), then there should be a propensity for ties to involve correct answers to other ties as learning proceeds.

\section{Method}

\section{Participants}

Twenty-four volunteers were recruited from the University of Saskatchewan Introductory Psychology participant pool and through advertisements around campus. Participant pool volunteers received two bonus class credits. All other participants were paid $\$ 15$ (Canadian). In general, incoming participants were alternately assigned to the consistent or inconsistent neighborhood conditions, but we deviated from this to maintain approximately equal proportions of men and women in the two conditions. Two participants in the inconsistent neighborhood condition were not at all successful in memorizing the pound arithmetic equations and were replaced. The final group of 24 included seven women and five men in the consistent condition and six women and six men in the inconsistent condition. Ages ranged from 18 to 31 years $($ mean $=22.9$ years $)$.

\section{Stimuli}

Stimuli were displayed as white characters in Courier New 12-pt font on a dark background. Equations to be memorized consisted of one of two sets of 16 pound arithmetic equations with digits 2 through 9 as operands. In each set, there were eight tie equations with repeated-digit operands (e.g., 2 \# 2 =65) and eight nontie equations with successive-digit operands in descending left-to-right order (e.g., 3 \# 2 = 75). Tie and nontie equations in adjacent rows and columns were neighbors in the pound arithmetic table (see Table 2).

In the consistent problem set, answers in adjacent columns and rows shared a common decade or unit value. In the inconsistent problem set, neighboring problems did not share a common decade or unit. Answers to both tie and nontie problems used digits in the range from 2 to 9 in the decade and unit positions. Answers ranged from 26 to 92 , had no consistent mathematical relationship to the operands, and were not significantly correlated with either operand or their sum or product [largest $r(14)=.22$, $p=.41]$. We tried to avoid equation or answer characteristics that would make individual equations distinctive: No answers were simple multiplication products (i.e., $2 \times 2=$ 4 to $9 \times 9=81$ ), answers were not decades (e.g., 30, 70) or

Table 2 Pound arithmetic table

\begin{tabular}{|c|c|c|c|c|c|c|c|c|}
\hline \multirow[b]{2}{*}{ Operands } & \multicolumn{8}{|c|}{ Operands } \\
\hline & 2 & 3 & 4 & 5 & 6 & 7 & 8 & 9 \\
\hline 2 & 65 & 47 & & & & & & \\
\hline 3 & 75 & 76 & 52 & & & \multirow{2}{*}{\multicolumn{3}{|c|}{$\begin{array}{l}\text { Tie and nontie neighbors } \\
\text { with inconsistent answers }\end{array}$}} \\
\hline 4 & & 26 & 29 & 83 & & & & \\
\hline 5 & & & 39 & 37 & 94 & & & \\
\hline 6 & & & & 47 & 43 & 39 & & \\
\hline 7 & \multicolumn{4}{|c|}{ Tie and nontie } & 83 & 84 & 26 & 34 \\
\hline 8 & \multicolumn{4}{|c|}{ neighbors with } & & 94 & 92 & 75 \\
\hline 9 & \multicolumn{4}{|c|}{ consistent answers } & & 34 & 52 & 58 \\
\hline
\end{tabular}

Answers to tie problems in bold. The larger operand for nontie problems always appeared on the left in both study and test blocks. The tie answers and nontie answers below the diagonal were used for the consistent neighborhood condition. The tie answers and nontie answers above the diagonal were used for the inconsistent neighborhood condition 
composed of a repeated digit (e.g., 33, 77), and answers did not contain either of the problem's operands.

The eight tie equations were identical in the consistent and inconsistent problem sets. The nontie equations involved the same operand pairs and answers in each set, but the answers were assigned to different nontie problems to achieve consistent or inconsistent relationships with neighboring ties. For example, in the consistent set, $6 \# 6=$ 43 was neighbored by $6 \# 5=47$ and $7 \# 6=83$, whereas in the inconsistent set, $6 \# 6=43$ was neighbored by $6 \# 5=$ 94 and $7 \# 6=39$. Note that although 94 and 39 share a common digit with 43 , the shared digit is not in the same decade or unit position. According to Verguts and Fias (2005a, 2005b), decade or unit consistency is the critical feature. Where possible in the consistent set, one neighbor had decade consistency (in the adjacent column), and the other had unit consistency (in the adjacent row). The problems $2 \# 2$, $9 \# 9$, and $9 \# 7$ had one consistent neighbor each. The nontie problem $9 \# 7=34$ necessarily had nonties as immediate neighbors and was included to create equal numbers of nontie and tie problems. The answer 34 shares a common unit with $7 \# 7=84$, but since 9 \# 7 and 7 \# 7 are not immediate neighbors, they would not be expected to strongly interact with one another (Verguts \& Fias, 2005b).

\section{Design and procedure}

Participants were tested individually during a 60-min session in a quiet, well-lit room with an experimenter present. Instructions and stimuli appeared on a highresolution monitor displayed by a Microsoft Windowsbased computer using E-prime v1.2 software. Participants sat about $60 \mathrm{~cm}$ from the monitor. Instructions explained that the participant's task was to memorize 16 pound arithmetic equations and that pound arithmetic bore no relation to standard arithmetic.

There were 14 study-test blocks. The participant pressed the spacebar to initiate the study and test phases in each block. Each study and test trial was preceded by a fixation dot that flashed twice within $1 \mathrm{~s}$ before the equation or problem appeared.

The study phase in each block consisted of the 16 pound arithmetic equations (e.g., $6 \# 5=94$ ) presented one at time in random order. Each equation appeared at the center of the screen for $3 \mathrm{~s}$. Then, the answer was replaced with a question mark ( 6 \# $5=$ ?), and a cursor appeared below to cue the participant to type in the answer, which was visible as it was typed. When a 2-digit number was entered, the correct equation was displayed at the center of the screen for $2.5 \mathrm{~s}$, with accuracy feedback displayed above it in the form of a green check mark for a correct response, or a red " $\times$ " for an incorrect response. If a 2-digit response was not entered within $5 \mathrm{~s}$, the correct equation appeared for $2.5 \mathrm{~s}$ at the center of the screen with the sentence, "Sorry, you took too long" presented above it.

After each study block, a 3-digit number between 100 and 120 appeared on the screen. Participants counted backward by threes out loud for $10 \mathrm{~s}$ until a screen prompt to press the spacebar appeared. The countdown served to reduce contributions of immediate short-term memory to the subsequent test block.

During the test phase, the 16 pound arithmetic problems were presented one at a time with a question mark (e.g., 6 \# $5=$ ?) in a random order. Participants had $5 \mathrm{~s}$ to type in a 2 digit answer. Response entry and feedback were identical to those in the study blocks. Response timing began when the problem appeared and stopped when participants typed the second digit of their proposed answer.

\section{Results}

\section{Percent correct}

Figure 1 presents the mean percentage of correct answers for pairs of successive test blocks as a function of neighborhood (consistent, inconsistent) and problem type (tie, nontie). Accuracy in the first two test blocks was less than $10 \%$ in all conditions. As Fig. 1 shows, accuracy increased steadily across blocks for all conditions, but a substantial advantage for the consistent neighborhood condition over the inconsistent condition emerged in Blocks 3 and 4. This advantage increased until the middle blocks and then remained relatively stable for the remainder of the experiment. This leveling off reflected the fact that some participants in the consistent condition were beginning to approach the $100 \%$ ceiling, whereas learning in the inconsistent condition lagged behind but was progressing. Eventually, of course, with sufficient motivated practice, the four functions would converge at $100 \%$ accuracy. Another obvious feature of Fig. 1 is that in both neighborhood conditions, an accuracy advantage for tie problems over nontie problems emerged after four or five blocks and persisted throughout the experiment. ${ }^{1}$

To confirm these observations statistically, we defined early practice as Blocks 1 to 7 and late practice as Blocks 8 to 14 , and performed a Practice (early, late) $\times$ Problem Type (tie, nontie) $\times$ Neighborhood (consistent, inconsistent) mixed-design ANOVA on participants' percentage correct in each cell. All ANOVA tests had $F$

\footnotetext{
${ }^{1}$ Overall, mean correct RT was faster later in practice $(2,317 \mathrm{~ms})$ than earlier in practice $(2,538 \mathrm{~ms})$; however, the relatively high errors rates prohibited a meaningful factorial analysis of RT for correct responses.
} 


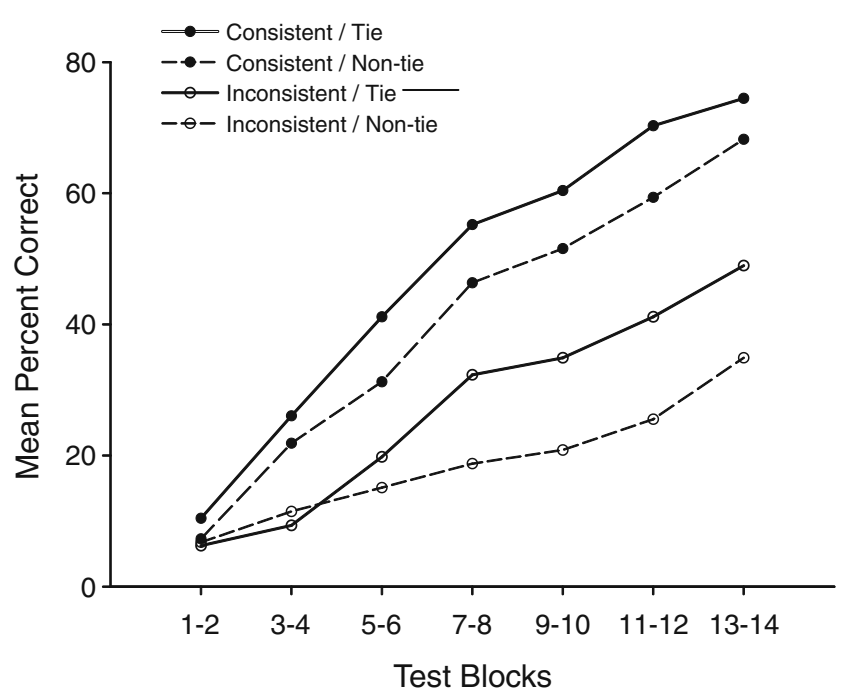

Fig. 1 Mean percentage of correct answers by neighborhood consistency, problem type, and test block

$(1,22)$ degrees of freedom. The corresponding means appear in Table $3 .^{2}$

The mean percentage of correct responses was, overall, much higher with consistent neighbors $(44.6 \%)$ than with inconsistent neighbors $(23.2 \%)[F(1,22)=11.0, M S E=$ 997.9, $p=.003]$. This confirms that neighborhood consistency greatly facilitated memorization of the equations. Participants' mean percentage of correct responses was, of course, higher later in practice $(47.8 \%)$ than early in practice $(19.9 \%)[F(1,22)=93.1, M S E=200.6]$, and the effects of practice interacted with neighborhood consistency $[F(1,22)=7.0, M S E=200.6, p=.015]$. Specifically, the advantage for consistency was smaller early in practice $(+13.7 \%)$ than later in practice $(+29.0 \%)$, confirming that the consistency advantage emerged as learning progressed. Percent correct was overall higher for tie problems $(37.8 \%)$ than for nontie problems $(29.9 \%)[F(1,22)=12.8, M S E=$ $116.2, p=.002]$, and this effect, too, emerged with practice: The tie advantage was smaller earlier in practice $(+4.7 \%)$ than later in practice $(+11.0 \%)[F(1,22)=4.6, M S E=51.4$, $p=.04]$.

We also conducted a problem-based Practice $\times$ Problem Type $\times$ Neighborhood multiple regression analysis of percent correct that treated type (tie, nontie) and neighborhood (consistent, inconsistent) as between-subjects (i.e., between-problem) factors and included neighbor distance

\footnotetext{
${ }^{2}$ We explored several different ways to define the practice factor for the ANOVAs. These all lead to essentially the same pattern of significant effects. We also performed parallel problem-based analyses. We do not report them fully here because the results were essentially identical to those of the participant-based analysis. There was no evidence in the problem-based data that either problem size (i.e., the numerical magnitude of the operands) or answer size affected performance.
}

Table 3 Mean percent correct, mean percent of neighbor errors, and percent of within-problem-type errors by neighborhood (consistent, inconsistent), practice(early, late), and problem type (ties, nonties)

\begin{tabular}{|c|c|c|c|c|c|c|}
\hline \multirow{2}{*}{$\begin{array}{l}\text { Test } \\
\text { Blocks }\end{array}$} & \multicolumn{3}{|c|}{ Consistent } & \multicolumn{3}{|c|}{ Inconsistent } \\
\hline & Ties & Nonties & All & Ties & Nonties & All \\
\hline \multicolumn{7}{|c|}{ Percent Correct } \\
\hline Early (1-7) & 30.2 & 23.4 & 26.8 & 14.4 & 11.8 & 13.1 \\
\hline Late $(8-14)$ & 66.4 & 58.3 & 62.4 & 40.3 & 26.3 & 33.3 \\
\hline \multicolumn{7}{|c|}{ Percent Neighbor Errors } \\
\hline Early (1-7) & 10.7 & 11.3 & 11.0 & 3.0 & 6.1 & 4.5 \\
\hline Late $(8-14)$ & 13.8 & 29.4 & 21.6 & 6.1 & 10.1 & 8.1 \\
\hline \multicolumn{7}{|c|}{ Percent of Within-Problem-Type Errors } \\
\hline Early (1-7) & 51.7 & 55.7 & 53.7 & 64.1 & 46.6 & 55.4 \\
\hline Late $(8-14)$ & 76.5 & 53.8 & 65.2 & 63.2 & 46.7 & 55.0 \\
\hline
\end{tabular}

(i.e., the mean difference between the correct answer and answers in the immediate neighboring row and column) as a covariate. The pattern of statistically significant effects was identical to the participant-based analysis, but there was also a significant effect of the distance covariate $[F(1$, $27)=6.5, M S E=165, p=.02]$. The regression coefficient relating average neighbor distance and percent correct was $-0.27 \%$ early in practice $[t(27)=-2.04, S E=0.13$, $p=.05]$ and $-0.40 \%$ late in practice $[\mathrm{t}(27)=-2.58, S E=$ $0.16, p=.02]$. This finding indicates that, over and above neighborhood-answer consistency, neighborhoodmagnitude consistency (i.e., having neighbors similar in numerical magnitude) also facilitated learning.

\section{Neighbor errors}

Most multiplication errors involve correct answers to other problems in the same times table (e.g., $5 \times 6=35$ ), predominantly immediate neighbors (i.e., the product obtained if either operand is changed by \pm 1 ; see, e.g., Campbell, 1999; Campbell \& Graham, 1985). By analogy to simple multiplication, we expected the proportion of neighbor errors (i.e., the correct answer if either operand is changed by \pm 1 ) in pound arithmetic to increase with practice; moreover, if neighbor errors are promoted by neighborhood consistency, as was proposed by Domahs et al. (2006), then neighbor errors should be promoted in the consistent condition relative to the inconsistent condition. All eight nontie problems had two immediate neighbors; consequently, we can estimate the chance probability of a neighbor error for nontie problems as 2/44 (4.5\%), where 44 represents the number of legitimate pound candidate answers between 23 and 98 (i.e., exclusive of decades, repeated digits, products from the standard times table, and a problem's correct answer). Six of the tie problems had 
two neighbors, and two had one neighbor (2\# 2 and $9 \# 9$ ); therefore, the expected rate on average was $1.75 / 44(4.0 \%)$ for ties.

Table 3 includes the mean percentage of errors that were neighbor errors. The observed grand mean percentage of neighbor errors was $11.3 \%(S E=1.2)$, more than double the estimated chance rates for ties or nonties. A Practice $\times$ Problem Type $\times$ Neighborhood ANOVA confirmed that the rate of neighbor errors was much higher later $(14.9 \%)$ than earlier in practice $(7.8 \%)[F(1,22)=18.2, M S E=66.3$, $p<.001]$. More important theoretically, the rate of neighbor errors also was much higher with a consistent neighborhood $(16.3 \%)$ than with an inconsistent neighborhood $(6.3 \%)[F$ $(1,22)=17.6, M S E=137.3, p<.001]$ (see also Domahs et al., 2006), and this difference was greater later $(+13.5 \%)$ than earlier $(+6.5 \%)$ in practice $[F(1,22)=4.5, M S E=$ $66.3, p=.05]$. The emergence of neighbor errors is a signature of the development of the operand-based organization of facts in memory (Campbell \& Graham, 1985); consequently, the results indicate that the development of the network structure for the pound arithmetic table was analogous to simple multiplication and was facilitated by neighborhood consistency.

Finally, although tie problems (e.g., 2 \# $2=65$ ) were learned faster than nonties $(2 \# 3=75)$, ties produced a lower percentage of neighbor errors (8.4\%) than did nonties $(14.2 \%)[F(1,22)=9.4, M S E=86.3, p=.006]$. This might appear to contradict our earlier claim that better learning should be associated with a higher proportion of neighbor errors. As will be shown next, however, there was a tendency for tie errors to involve answers to other tie problems, which worked against neighbor errors for ties (i.e., ties' neighbors were always nontie problems).

\section{Within-problem-type errors}

Nonneighbor errors involving a correct answer to another problem in the pound table (henceforth table errors) provided an opportunity to determine whether tie and nontie problems are categorically distinct in memory. If they are, then errors would be expected to be more likely to originate from within the same problem type (Campbell, 1995). This question could not be addressed with neighbor errors because in our stimulus sets, these always involved an answer originating from the other problem type. In contrast, other table errors could originate from either the tie answer set or the nontie answer set.

Given random selection of a table error, then for any nontie problem, the chance probability that it would be a within-type error was $7 / 13(53.8 \%)$, with 7 being the number of different within-type errors possible (i.e., the set of incorrect nontie answers) and 13 being the number of different possible table errors for each nontie problem (i.e.,
16 pound answers in total, minus a problem's correct answer and two neighbors). For tie problems, the average chance rate of a within-type error was 7/13.25 (52.8\%), with seven being the number of possible within-type errors (i.e., incorrect tie answers), and 13.25 being the average number of different possible table errors for tie problems (16 minus the correct answer and 1.75 neighbors on average).

One participant in each neighborhood condition made no nonneighbor table errors in at least one cell. The mean percentage of within-type table errors as a function of Practice $\times$ Problem Type $\times$ Neighborhood for the remaining 11 participants in each group appear in Table 3. The corresponding ANOVA indicated only a main effect of problem type $[F(1,20)=7.7, M S E=494.0, p=.01$; all other $p \mathrm{~s}>.15]$. For tie problems, $63.9 \%$ of table errors on average were within-type errors (i.e., answers to other tie problems), well above the estimated chance rate of $52.8 \%[t(21)=3.5, S E=3.2, p=.002]$. In contrast, on average only $50.7 \%$ of nontie table errors were withintype errors (i.e., answers to other nontie problems), which was not different from the estimated chance rate of $53.8 \%$ $[t(21)=-0.8, S E=4.1, p=.46]$. The bias for tie-answer errors on tie problems would work against neighbor errors, which always involved nontie answers. In the consistent neighborhood condition, the rate of tie-answer errors on tie problems was $77 \%$ late in practice relative to the $53 \%$ expected by chance. This strong bias would explain why the ties, relative to the nonties, presented a substantially lower rate of neighbor errors late in practice in the consistent condition ( $14 \%$ vs. $29 \%$; see Table 2$)$.

\section{Discussion}

The pound arithmetic experiment was designed to investigate effects of answer neighborhood consistency on memory for number facts. According to the IN model (Verguts \& Fias, 2005a; 2005b), when categorically related problems (i.e., problems sharing a common operand) have neighbors with common decade or unit answer elements, this answer consistency tends to facilitate learning and memory, whereas inconsistent neighbors tend to inhibit learning. The present experiment showed that answer consistency greatly facilitated learning and memory of pound arithmetic facts. A substantial accuracy advantage for the consistent neighborhood condition over the inconsistent condition emerged after only two blocks of practice and persisted through the 14 study-test cycles. By the end of the experiment, mean accuracy was $71 \%$ for the consistent neighborhood condition as compared with only $42 \%$ for the inconsistent neighborhood condition. The problem-based analyses showed, furthermore, that over 
and above neighborhood answer consistency, learning was facilitated when the average numerical difference between the correct answer and neighboring answers was smaller rather than larger. Thus, both neighborhood answer-element consistency and neighborhood answer-magnitude consistency facilitated memorization of the pound equations.

There was also a robust accuracy advantage for tie problems relative to nontie problems in both neighborhood conditions. The magnitude of the tie-problem advantage on accuracy was, overall, the same with consistent and inconsistent neighbors. In the IN model, an advantage for tie problems (e.g., $3 \times 3,5 \times 5$ ) occurs because the tieanswer neighborhoods are relatively more consistent (or, equivalently, less inconsistent) than nontie answer neighborhoods (e.g., $3 \times 4 ; 4 \times 5$ ). In other words, it is the contrasting levels of consistency that are assumed to produce the tie effect. Our experiment showed a learning advantage for ties regardless of consistency; thus, the tie effect in pound arithmetic did not depend on neighborhood consistency.

An analysis of operand-related errors provided a window on the development of the memory network for pound arithmetic. In simple multiplication, operand-related errors (e.g., $3 \times 9=18 ; 6 \times 9=63$ ) are assumed to reflect the factor-based categorical structure of the memory network for multiplication facts; furthermore, such errors are predominantly immediate neighbors. Consequently, if memorization of the pound arithmetic table involved processes analogous to simple multiplication, then we expected neighbor errors to occur above chance rates and to account for an increasing percentage of errors as the memory network developed. Indeed, the overall rate of neighbor errors was well above chance and was approximately twice as high later (14.9\%) than earlier in practice (7.8\%). This confirms that memory for the pound arithmetic table involved an operand-based categorical structure analogous to simple multiplication.

Most importantly, however, the rate of neighbor errors was overall much higher with a consistent answer neighborhood $(16.3 \%)$ than with an inconsistent answer neighborhood $(6.3 \%)$, and this difference was greater later $(+13.3 \%)$ than earlier in practice $(+6.5 \%)$. This finding reinforces the conclusions of Domahs et al. (2006) that operand-related errors are promoted by neighborhood consistency. Indeed, the results provide direct evidence that the development of the operand-based memory network for pound arithmetic was facilitated by neighborhood consistency and strongly support the IN model's central assumption that neighborhood consistency can contribute directly to the learning and development of memory for arithmetic facts.

The analysis of nonneighbor table errors revealed another structural feature of memory for pound arithmetic. These errors allowed us to assess whether tie and nontie problems were categorically distinct in memory by analyzing the probability that table errors originated within the same problem type (Campbell, 1995). Neighbor errors did not afford this analysis because they always involved an answer associated with the other problem type. For tie problems, the rate of table errors involving answers to other tie problems was $64 \%$, well above the rate expected by chance. This finding suggests that tie problems, in addition to activating neighbor answers, also activated answers to other tie problems or inhibited answers to nonties. This finding is consistent with the proposal that ties may be represented as a distinct category of problems (Campbell, 1995). In contrast, errors on nontie problems presented no such bias, with tie and nontie answers equally likely to appear among table errors. This categorical asymmetry between ties and nonties would contribute to the learning and performance advantage for ties. For nonties, the candidate set of answers included answers to all other problems regardless of problem type. For ties, however, the candidate set became more focused on the set of tie answers, which would effectively reduce the number of competing answers and facilitate learning. The emergence of tie equations as a distinct subcategory may occur because they all share a distinctive feature (i.e., a twinned operand) that distinguishes them from the nonties (see also Lebiere \& Anderson, 1998).

\section{Origins of neighborhood consistency effects}

What is the mechanism of consistency effects in numberfact memory? Verguts and Fias (2005a, 2005b) drew an analogy to neighborhood effects in single-word reading: Words that share a regular pronunciation with orthographic neighbors (e.g., fade has consistent neighbors grade, jade, made) are pronounced more quickly than words with few consistent neighbors or words with an inconsistent neighborhood that includes an irregular pronunciation (e.g., wave has the inconsistent neighbor have; see, e.g., Glushko, 1979). Neighborhood effects in word reading typically are attributed to orthographic-phonological consistency (Andrews, 1997; but see Mulatti, Reynolds, \& Besner, 2006), but, unlike single-word reading, production of a multiplication answer given a visually presented problem does not entail an orthographic-phonological mapping (e.g., generating " 18 " given $2 \times 9$ ). Consequently, it is unlikely that the same mechanism mediates neighborhood effects in singleword reading and number-fact memory.

Instead, we propose that answer consistency effects arise from mechanisms of associative memory, particularly associative interference owing to each problem operand (e.g., the factor 7) being associated with multiple products $(14,21,28,35$, etc.). This one-to-many associative structure interferes with learning and retrieval performance 
(Campbell, 1995; Lebiere \& Anderson, 1998; see also Pirolli \& Anderson, 1985). When a problem is presented, a semantic neighborhood of operand-related products is activated, and retrieval of the correct product must overcome interference from its neighbors. According to Phenix and Campbell (2004), this interference is resolved by competitor inhibition, as evidenced by retrieval-induced forgetting of neighboring products. Furthermore, competitors attract more inhibition when their competition is relatively strong (Campbell \& Phenix, 2009; Levy \& Anderson, 2002). From this perspective, the present findings with pound arithmetic suggest that competitor interference is reduced when neighboring products share a common decade or unit component. One possibility, consistent with the IN model, is that associative competition and inhibition between multiplication facts occurs independently for the decade and unit components of their answers. Decade or unit consistency would reduce interference because a consistent neighbor is not a competitor at the decade or unit level and would attract less inhibition than an entirely inconsistent neighbor answer.

Relevance of pound arithmetic to multiplication and the interacting neighbors model

Unlike the real multiplication table, there was no underlying arithmetic operation relating operands to answers in the pound table. Given this consideration, how relevant is pound arithmetic to actual multiplication and the IN model? The IN model makes no assumptions regarding mathematical meaning. Indeed, we sought to eliminate any obvious numerical relation between pound operands and answers in order to isolate structural features of the multiplication tables deemed to be central to multiplication memory in the IN model: The pound problems were composed of pairs of single-digit operands from a continuous sequence (i.e., 2 to 9), all operands appeared in multiple problems, there was a wide range of answers (26 to 95), and the pound table included both tie and nontie problems. These analogous features of the pound table gave rise to phenomena that directly paralleled real multiplication, including the early emergence of neighbor errors in the consistent neighborhood condition, and a learning advantage for tie problems relative to nontie problems. Within this context, pound arithmetic also provided clear evidence for a learning advantage with consistent neighbors relative to inconsistent neighbors.

We found no evidence of a problem-size effect in our problem-based analysis, but this too is consistent with the IN model: With practice frequency held constant, the IN model predicts a problem-size effect (i.e., increased difficulty as problem size increases) only if neighborhood consistency is negatively correlated with problem size, as it is in real multiplication (Verguts \& Fias, 2005a). In our pound arithmetic table, problem size and consistency were uncorrelated, and there was no evidence of a problem-size effect on accuracy as would be expected according to the IN model. Future research with pound arithmetic could manipulate the correlation between problem size and consistency to demonstrate a standard problem-size effect when the correlation is negative, or a reverse problem-size effect if the correlation is positive.

One feature of our results not predicted by the IN model, however, was the facilitative effect on learning of neighbormagnitude consistency (i.e., the average nearness of adjacent neighbors) over and above the effects of neighbor-answer consistency. The magnitude of answers, per se, appears to play no role in the IN model presented in Verguts and Fias (2005a). Our finding suggests, however, that participants encoded answers as meaningful quantities and that local semantic coherence among neighbors facilitated memory. Thus, although there was no systematic relation between operands and pound answers globally, participants encoded local semantic regularities into the developing network representation. This highlights the possibility that when there is a global relation between operand and answer magnitude as in real arithmetic, learners exploit the semantic coherence afforded by this regularity. This novel finding attests further to the relevance of pound arithmetic to learning and memory of arithmetic facts.

\section{Conclusions}

According to the IN model (Verguts \& Fias, 2005a), major features of simple multiplication performance, including the problem size and the performance advantage for fives and ties, all reflect the effect of neighborhood answer consistency. Here, memorization of the pound arithmetic table presented a robust effect of neighborhood consistency, with the rate of learning almost doubling that of the inconsistent condition across the 14 study-test cycles. Consistency facilitated development of an operand-based categorical structure in memory analogous to simple multiplication, as evidenced by the early emergence of neighbor errors in the consistent condition. The results thereby provide strong empirical support for the potential role of neighborhood consistency in memorization of multiplication facts as proposed by Verguts and Fias (2005a, 2005b). This was a unique prediction of the IN model, and no other model of number fact memory, nor any more general model of associative memory that we are familiar with, addresses this type of neighborhood consistency effect. Consequently, apart from informing research in cognitive arithmetic, the robust effects of neighborhood consistency observed in the 
present study are potentially relevant to memory researchers more generally.

\section{References}

Andrews, S. (1997). The effect of orthographic similarity on lexical retrieval: Resolving neighborhood conflicts. Psychonomic Bulletin \& Review, 4, 439-461.

Ashcraft, M. H. (1992). Cognitive arithmetic: A review of theory and data. Cognition, 44, 75-106. doi:10.1016/0010-0277(92)90051-I

Campbell, J. I. D. (1994). Architectures for numerical cognition. Cognition, 53, 1-44. doi:10.1016/0010-0277(94)90075-2

Campbell, J. I. D. (1995). Mechanisms of simple addition and multiplication: A modified network-interference theory and simulation. Mathematical Cognition, 1, 121-165.

Campbell, J. I. D. (1997). On the relation between skilled performance of simple division and multiplication. Journal of Experimental Psychology. Learning, Memory, and Cognition, 23, 1140-1159. doi:10.1037/0278-7393.23.5.1140

Campbell, J. I. D. (1999). Division by multiplication. Memory \& Cognition, 27, 791-802.

Campbell, J. I. D., \& Graham, D. J. (1985). Mental multiplication skill: Structure, process, and acquisition. Canadian Journal of Psychology, 39, 338-366. doi:10.1037/h0080065

Campbell, J. I. D., \& Gunter, R. (2002). Calculation, culture, and the repeated operand effect. Cognition, 86, 71-96. doi:10.1016/ S0010-0277(02)00138-5

Campbell, J. I. D., \& Metcalfe, A. W. S. (2007). Numeral format and arithmetic rules. European Journal of Cognitive Psychology, 19, 335-355. doi:10.1080/09541440600717610

Campbell, J. I. D., \& Phenix, T. L. (2009). Target strength and retrieval-induced forgetting in semantic recall. Memory \& Cognition, 37, 65-72. doi:10.3758/MC.37.1.65

Domahs, F., Delazer, M., \& Nuerk, H. (2006). What makes multiplication facts difficult: Problem-size or neighborhood consistency? Experimental Psychology, 53, 275-282. doi:10.1027/1618-3169.53.4.275

Galfano, G., Rusconi, E., \& Umiltà, C. (2003). Automatic activation of multiplication facts: Evidence from the nodes adjacent to the product. The Quarterly Journal of Experimental Psychology, $56 A, 31-61$.

Glushko, R. J. (1979). The organization and activation of orthographic knowledge in reading aloud. Journal of Experimental Psychology: Human Perception and Performance, 5, 674-691. doi:10.1037/0096-1523.5.4.674

Koshmider, J. W., \& Ashcraft, M. H. (1991). The development of children's mental multiplication skills. Journal of Experimental Child Psychology, 51, 53-89. doi:10.1016/0022-0965(91)90077-6

Lebiere, C., \& Anderson, J. R. (1998). Cognitive arithmetic. The atomic components of thought. Mahwah, NJ: Erlbaum.

LeFevre, J., Bisanz, J., Daley, K. E., Buffone, L., Greenham, S. L., \& Sadesky, G. S. (1996). Multiple routes to solution of single-digit multiplication problems. Journal of Experimental Psychology: General, 125, 284-306. doi:10.1037/0096-3445.125.3.284

Levy, B. J., \& Anderson, M. C. (2002). Inhibitory processes and the control of memory retrieval. Trends in Cognitive Sciences, 6, 299-305. doi:10.1016/S1364-6613(02)01923-X

McCloskey, M., \& Lindemann, A. M. (1992). MATHNET: Preliminary results from a distributed model of arithmetic fact retrieval.
In J. I. D. Campbell (Ed.), The nature and origins and mathematical skills (pp. 365-409). Amsterdam: Elsevier. doi:10.1016/S0166-4115(08)60892-4

Mulatti, C., Reynolds, M. G., \& Besner, D. (2006). Neighborhood effects in reading aloud: New findings and new challenges for computational models. Journal of Experimental Psychology: Human Perception and Performance, 32, 799-810. doi:10.1037/0096-1523.32.4.799

Norem, G. M., \& Knight, F. B. (1930). The learning of the one hundred multiplication combinations. National Society for the Study of Education: Report on the Society's Committee on Arithmetic, 15, Yearbook 29, 551-567.

Nuerk, H.-C., Weger, U., \& Willmes, K. (2001). Decade breaks in the mental number line? Putting the tens and units back in different bins. Cognition, 82, B25-B33. doi:10.1016/S0010-0277(01)00142-1

Phenix, T. L., \& Campbell, J. I. D. (2004). Effects of multiplication practice on product verification: Integrated structures model or retrieval induced forgetting? Memory \& Cognition, 32, 324335.

Pirolli, P., \& Anderson, J. R. (1985). The role of practice in fact retrieval. Journal of Experimental Psychology. Learning, Memory, and Cognition, 11, 136-153. doi:10.1037/02787393.11.1.136

Ratinckx, E., Brysbaert, M., \& Fias, W. (2005). Naming two-digit arabic numerals: Evidence from masked priming studies. Journal of Experimental Psychology: Human Perception and Performance, 31, 1150-1163. doi:10.1037/0096-1523.31.5.1150

Rickard, T. C. (2005). A revised identical elements model of arithmetic fact representation. Journal of Experimental Psychology. Learning, Memory, and Cognition, 31, 250-257. doi: $10.1037 / 0278-7393.31 .2 .250$

Rickard, T. C., Healy, A. F., \& Bourne, L. E., Jr. (1994). On the representation of arithmetic facts: Operand order, symbol, and operation transfer effects. Journal of Experimental Psychology. Learning, Memory, and Cognition, 20, 1139-1153. doi:10.1037/ 0278-7393.20.5.1139

Robert, N. D., \& Campbell, J. I. D. (2008). Simple addition and multiplication: No comparison. European Journal of Cognitive Psychology, 20, 123-138. doi:10.1080/09541440701275823

Siegler, R. S. (1988). Strategy choice procedures and the development of multiplication skill. Journal of Experimental Psychology: General, 117, 258-275. doi:10.1037/0096-3445.117.3.258

Verguts, T., \& Fias, W. (2005a). Interacting neighbors: A connectionist model of retrieval in single-digit multiplication. Memory \& Cognition, 33, 1-16.

Verguts, T., \& Fias, W. (2005b). Neighbourhood effects in mental arithmetic. Psychology Science, 47, 132-140.

Zbrodoff, N. J., \& Logan, G. D. (2005). What everyone finds: The problem-size effect. In J. I. D. Campbell (Ed.), Handbook of mathematical cognition (pp. 331-346). New York: Psychology Press.

This research was supported by a grant from the Natural Sciences and Engineering Research Council of Canada. The authors thank Sean Sacher for assistance in data collection. Address correspondence to Jamie Campbell, Department of Psychology, 9 Campus Drive, University of Saskatchewan, Saskatoon, SK, Canada, S7N 5A5 (e-mail: jamie.campbell@usask.ca). 